\title{
Rho decay width from the lattice
}

\author{
J. Frison ${ }^{* a}$, S. Durr ${ }^{b, c}$, Z. Fodor ${ }^{b, c, d}$, C. Hoelbling ${ }^{b}$, S.D. Katz ${ }^{b, d}$, S. Krieg ${ }^{b, c}$, T. Kurth ${ }^{b}$, \\ L. Lellouch ${ }^{a}$, T. Lippert ${ }^{b, c}$, A. Portelli ${ }^{a}$, A. Ramos ${ }^{a}$ and K.K. Szabo ${ }^{b}$ \\ (Budapest-Marseille-Wuppertal Collaboration) \\ ${ }^{a}$ Centre de Physique Théorique $\dagger$, Case 907, CNRS Luminy, F-13288 Marseille Cedex 9, France. \\ ${ }^{b}$ Bergische Universität Wuppertal, Gaussstr. 20, D-42119 Wuppertal, Germany. \\ ${ }^{c} J u ̈ l i c h$ Supercomputing Centre, Forschungszentrum Jülich, D-52425 Jülich, Germany. \\ ${ }^{d}$ Institute for Theoretical Physics, Eötvös University, H-1117 Budapest, Hungary. \\ E-mail: frisonecpt.univ-mrs.fr
}

While the masses of light hadrons have been extensively studied in lattice QCD simulations, there exist only a few exploratory calculations of the strong decay widths of hadronic resonances. We will present preliminary results of a computation of the rho meson width obtained using $N_{f}=2+1$ flavor simulations. The work is based on Lüscher's formalism and its extension to moving frames.

The XXVIII International Symposium on Lattice Field Theory

June 14-19,2010

Villasimius, Sardinia Italy

\footnotetext{
* Speaker.

${ }^{\dagger}$ CPT is research unit UMR 6207 of the CNRS and of the universities Aix-Marseille II, Aix-Marseille I and Sud Toulon-Var, and is affiliated with the FRUMAM.
} 
The light hadron masses have been extensively and quite successfully studied in lattice QCD simulations [1,2]. However, the study of strong decays remains a challenge that only a few exploratory calculations have addressed so far $[3,4]$.

Until recently this issue could be ignored: firstly because the sea quarks required for a multibody decay are not present in the quenched approximation, and then because energy conservation leaves little or no phase space in those decays for unphysically large light quark masses. But as we get closer to the physical point and improve our precision, this issue has to be addressed for us to be able to reliably determine masses of resonant states. Additionally, it is an exciting way to test the characteristic dynamical effects of sea quarks.

A formalism waq developped by Lüscher [5-9], and extended to moving frames [10], which describes the modification of the finite-volume quantization condition of scattering-state momenta under interactions. We use this framework to compute the rho decay width on a subset of the Budapest-Marseille-Wuppertal collaboration configurations [12,13], which features improved Wilson fermions with $N_{f}=2+1$ flavors of sea quarks.

\section{Introducing the two-pions levels}

\subsection{Basics of avoided crossings}

If $\rho$ were not coupled to $\pi \pi$, the spectrum as a function of box size $L$ would only consist of :

- a $\rho$ state, whose energy would be a constant $m_{\rho}$ up to exponentially suppressed finite-volume corrections [5], and

- free two-pion states, whose momenta $\vec{k}=(2 \pi / L) \vec{n}$, and energies $E=\sqrt{m_{\pi}^{2}+\vec{k}^{2}}$ are quantized.

For some particular box sizes the $\rho$ states crosses one of the free two-pions states. At these points we would have a degeneracy but nothing particular would happen. But if now we turn on interactions, the situation becomes very different: we know from quantum mechanics that two eigenstates cannot cross. The interaction mixes $\rho$ and $\pi \pi$ into new eingenstates which exhibit an avoided level crossing phenomenon (Fig. 1). These avoided crossings therefore contain information on the coupling between the states, and hence on the $\rho$ width.

\subsection{Lüscher's Formula}

In a finite box we expect the pions to interact then propagate over small distance, then interact again, and so on. This can be represented as an expansion in a series of two-particles-irreducible kernels as shown in Fig. 2. When this expansion is resummed, the interactions move $F$ poles (free two-pions states) to $1-M F$ zeros [11]. The zeros of $1-M F$ correspond to the solutions of Lüscher's formula, which was first derived in [6].

Lüscher's formula is only valid below the $4 \pi$ inelastic threshold. Expressed in a form exhibiting its role of quantization condition, it reads (up to exponentially small terms in $m_{\pi} L$ ):

$$
\Phi(q)=n \pi-\delta(q)
$$




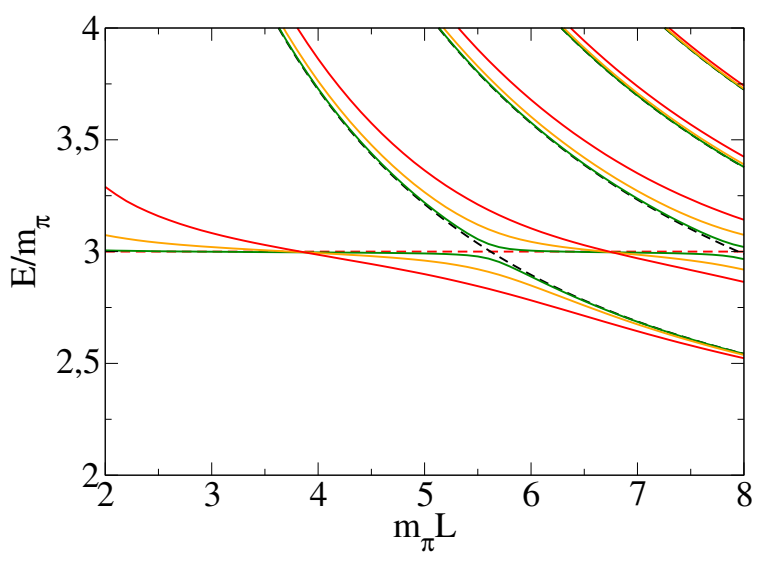

Figure 1: Finite-volume spectrum in the $I=J=1$ channel as a function of $m_{\pi} L$, for $m_{\rho} / m_{\pi}=3$, neglecting exponentially suppressed corrections. The dash lines depict the free spectrum and the solid lines show its deformation with increasing $\pi \pi$ - $\rho$ coupling $g$, from green to red. (The model used is described in Sec. 1.3.)

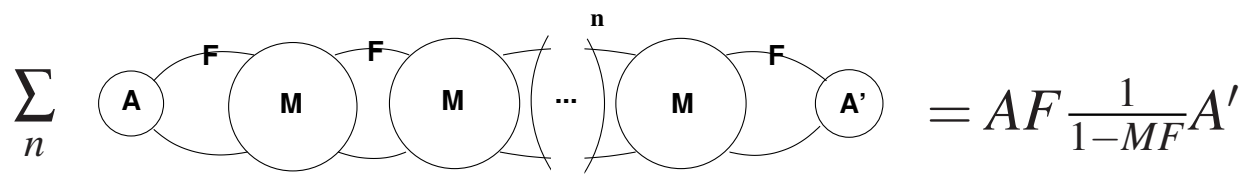

Figure 2: Here we represent the finite-volume correction of an arbitrary correlator, computed under the inelastic threshold up to exponentially small corrections. $M$ is the infinite-volume amputated four-point function, while $F$ is the difference between finite-volume loops and infinite-volume ones. $M$ is obtained as a resummation of a volume-independant series of 2-particle-irreducible kernels.

using the reduced momentum $q=k L / 2 \pi$ and the $\delta$ phase shift in the $I=J=1$ channel. $\Phi$ is a known kinematical function of $q$, expressing the breaking of rotational invariance by the cubic box [8].

\subsection{The $g$-coupling model}

Lüscher's equation gives us the phase shift for a few discrete values of the momentum, determined by the parameters of the simulation. It would be difficult and very costly numerically to significantly increase the number of such momenta. Therefore we need a model to reconstruct the phase shift from a few points. Following [9] we use both an effective range approximation and an effective lagrangian $\mathscr{L}_{\text {eff }}=g \varepsilon_{a b c} \rho_{\mu}^{a} \pi^{b} \partial^{\mu} \pi^{c}$.

The effective range approximation parametrizes the phase shift in terms of a Taylor expansion around $k_{\rho}=\sqrt{E^{2} / 4-m_{\pi}^{2}}$, which is is the momentum that $\rho^{\prime} s$ decay products would have in infinite-volume:

$$
\frac{k^{3}}{W} \cot \delta=b\left(k^{2}-k_{\rho}^{2}\right)
$$

The second ingredient allows us to parametrize the coupling of the $\rho$ to $\pi \pi$ states in terms of a constant coupling $g$ that should have a rather small dependance on the quark mass. On the other 
hand the width $\Gamma_{\rho}$ is strongly dependant on kinematics:

$$
\Gamma_{\rho}=\frac{g^{2}}{6 \pi} \cdot \frac{k_{\rho}^{3}}{m_{\rho}^{2}} .
$$

Using experimental values for the masses and the width (1.3) yields $g \simeq 6.0$ [9].

\section{Results with a single interpolating operator}

The formulae (1.2) and (1.3) can be combined into a very simple form, giving the correction to get the $\rho$ mass from the energy $E$ of an arbitrary eigenstate in the $I=J=1$ channel:

$$
m_{\rho}^{2}=E^{2}-\frac{g^{2}}{6 \pi} \frac{k^{3}}{E} \cot \Phi(k L / 2 \pi) \quad \text { where } k=\sqrt{\frac{E^{2}}{4}-m_{\pi}^{2}}
$$

In this expression, the unknowns are $m_{\rho}$ and $g$. Instead of using two energy levels at a given quark mass, one can extract the ground state energy level at different quark masses. But the kinematics required in that case are contradictory: on the one hand we would get no signal for the width if the state is far below the first free two-pion state; on the other hand, near the first crossing one cannot disentangle the contributions of the $\rho$ mass and width to the measured energy level.

In [1] we considered only situations on which the ground state obtained with a single operator is far from the first crossing. Thus, in combined fits of our 6-stout data, we obtained a precise determination of $m_{\rho}\left(m_{\rho} \sim 5 \%\right)$, but a much less precise determination of $g: g=9.5 \pm 4.6$.

\section{Using two interpolating operators}

\subsection{Generalized Eigenvalue Problem}

We now briefly present the principle of extraction of several energies using a variational method [7]. Let us first assume that we are computing observables only affected by $N$ eigenstates. We show that the $N$ energies can be computed from an $N \times N$ matrix of cross-correlators

$$
C_{i j}(t)=\left\langle 0\left|\mathscr{O}_{i}(t) \mathscr{O}_{j}(0)\right| 0\right\rangle, \quad \text { with } i, j=1 \ldots N .
$$

We first decompose them on the energy eigenstates and express them in a compact matrix form:

$$
\begin{aligned}
C_{i j}(t) & =\sum_{n}\left\langle 0\left|\mathscr{O}_{i}\right| n\right\rangle e^{-E_{n} t}\left\langle n\left|\mathscr{O}_{j}\right| 0\right\rangle \\
& =\sum_{m, n}\left(V^{\dagger}\right)_{i, m} \cdot D_{m, n}(t) \cdot V_{n, j},
\end{aligned}
$$

so $V_{n, j}$ is the $n$ th-state content of the $\mathscr{O}_{i}$ operator and $D$ is a diagonal matrix containing the exponentials of the energies. Now we immediatly see that $C(t) C^{-1}\left(t_{0}\right)$ is diagonal in the eigenbasis:

$$
C(t) C^{-1}\left(t_{0}\right)=V^{\dagger} D(t) V\left(V^{\dagger} D\left(t_{0}\right) V\right)^{-1}=\left(V^{\dagger}\right) D\left(t-t_{0}\right)\left(V^{\dagger}\right)^{-1},
$$

and its eigenvectors give the energies through:

$$
\lambda_{i}=e^{-E_{i}\left(t-t_{0}\right)} .
$$




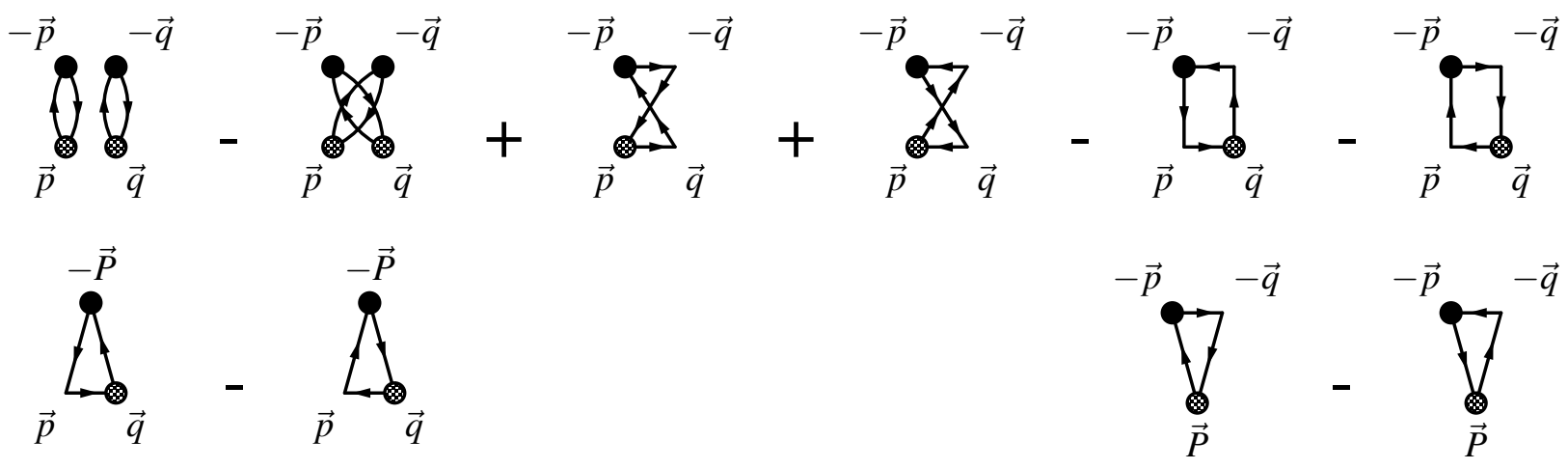

Figure 3: The contractions of $\pi \pi \rightarrow \pi \pi$ (top), $\pi \pi \rightarrow \rho$ (bottom-left) and $\rho \rightarrow \pi \pi$ (bottom-right), the $\rho \rightarrow \rho$ being trivial. Time flows upward from 0 to $t$. Black dots represent an explicit summation whereas shaded dots represent a noise-noise contact. Between those dots we can have $Q$ propagators (one line) or $W$ propagator (two lines).

This is exact if and only if the operators are linearly independent (so we can invert the $V \mathrm{~s}$ ). Note that in the case $N=1$ this is simply the effective mass method.

In practice we can only compute the matrix of operators obtained from a few $N$ operators. Thus only the $N$ low-lying levels are asymptotically known, and higher levels are treated as a contamination at short times. The energies are computed with errors of order $\exp \left[\left(E_{N}-E_{N+1}\right) t\right]$ and $\exp \left[\left(E_{N}-E_{N+1}\right) t_{0}\right]$, so $t$ and $t_{0}$ must be large $\left(t-t_{0}\right.$ is not very important, since it only enters in polynomial prefactors). A more precise study of higher-level corrections on some derived quantities is made in [7].

\subsection{Operators and contractions}

To implement the generalized eigenvalue approch described above, we consider two operators. The first is the point $\rho$ meson operator

$$
\rho_{i}=\bar{u} \gamma_{i} u-\bar{d} \gamma_{i} d
$$

The second operator is constructed from two, local pion-operators, to give it a better coupling to scattering states:

$$
\pi \pi_{i}(\vec{p}, \vec{q})=\left(p_{i}-q_{i}\right)\left[\pi^{+}(\vec{p}) \pi^{-}(\vec{q})-\pi^{-}(\vec{p}) \pi^{+}(\vec{q})\right] .
$$

Then, the cross-correlator is computed by contracting stochastic propagators. Following [3] we use two kinds of propagators:

$$
\begin{aligned}
Q\left(\vec{x}, t \mid \vec{q}, t_{s}, \xi_{j}\right) & =\sum_{\vec{y}} D^{-1}\left(\vec{x}, t ; \vec{y}, t_{s}\right) \cdot\left[e^{i \vec{p} \cdot \vec{y}} \xi_{j}(\vec{y})\right], \\
W\left(\vec{x}, t\left|\vec{k}, t_{1}\right| \vec{q}, t_{s}\right) & =\sum_{\vec{z}} D^{-1}\left(\vec{x}, t ; \vec{z}, t_{1}\right) \cdot\left[e^{i \vec{k} \cdot \vec{z}} \gamma_{5} Q\left(\vec{z}, t_{1} \mid \vec{q}, t_{s}\right)\right],
\end{aligned}
$$

where the $\xi_{j}(\vec{y})$ are three-dimensional, random $U(1)$ sources. The contractions are described in Fig. 3, and here we make explicit the first one :

$$
G_{\pi \pi \rightarrow \rho}^{1 s t}=\sum_{j, \vec{x}} e^{-i \vec{P} \cdot \vec{x}}\left\langle Q\left(\vec{x}, t \mid \overrightarrow{0}, t_{s}, \xi_{j}\right) W^{\dagger}\left(\vec{x}, t\left|-\vec{p}, t_{s}\right|-\vec{q}, t_{s}, \xi_{j}\right) \gamma_{5} \gamma_{3}\right\rangle .
$$




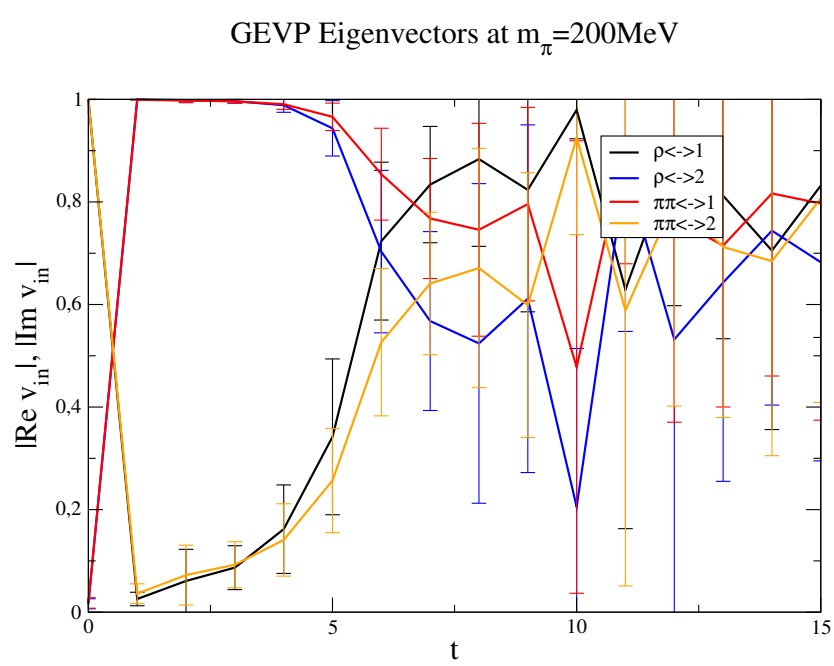

Figure 4: Components of generalized eigenvectors, normalized and obtained from operators normalized so that $\langle 0|\mathscr{O}(1) \mathscr{O}(0)| 0\rangle=1$. The eigenvectors are very sensitive to both higher-level contamination and statistical errors. For $t<6$ higher-state contamination is obvious, but for $6 \leq t \leq 9$ or even more the eigenvectors are constant as should be.

\section{Preliminary results}

We use the simulation setup of the Budapest-Marseille-Wuppertal collaboration [1,14] with 2 levels of HEX smearing [12,13,15], featuring $N_{f}=2+1$ flavors of tree-level improved Clover fermions [14] and the tree-level improved Lüscher-Weisz gauge action [16]. We choose two simulations, for which the two lowest-lying scattering states are near the crossing.

The first point is for $m_{\pi} \simeq 200 \mathrm{MeV}$, with $\beta=3.31$ and $a=0.116 \mathrm{fm}$ on a $32^{3} \times 48$ lattice. Contractions were computed in the center-of-mass frame $\vec{P}=(0,0,0)$, in which the $\rho$ mass nearly crosses the $\pi(0,0,2 \pi / L) \pi(0,0,-2 \pi / L)$ free two-pion state.

The other point has $m_{\pi} \simeq 340 \mathrm{MeV}$, with $\beta=3.31$ and $a=0.116 \mathrm{fm}$. Here, the lattice size is $24^{3} \times 48$. The analysis is performed in the moving frame $\vec{P}=(0,0,2 \pi / L)$, in which the $\rho$ can nearly "decay" into $\pi(0,0,2 \pi / L) \pi(0,0,0)$. and 5):

Using Eq. (2.1) on the two energies obtained with the variational method, we get (see Fig. 4

$$
\begin{array}{ll}
g=5.5 \pm 2.9 & \text { for the } m_{\pi} \simeq 200 \mathrm{MeV} \text { point, } \\
g=6.6 \pm 3.4 & \text { for the } m_{\pi} \simeq 340 \mathrm{MeV} \text { point }
\end{array}
$$

where the error is purely statistical. Combining the two results, we obtain the very preliminary estimate

$$
g=6.0 \pm 2.2 \text { (stat.), }
$$

which agrees with the experimental value given after Eq. (1.3).

\section{Aknowledgments}

Computations were performed using HPC resources from GENCI-[CCRT/IDRIS] (grant 52275), 


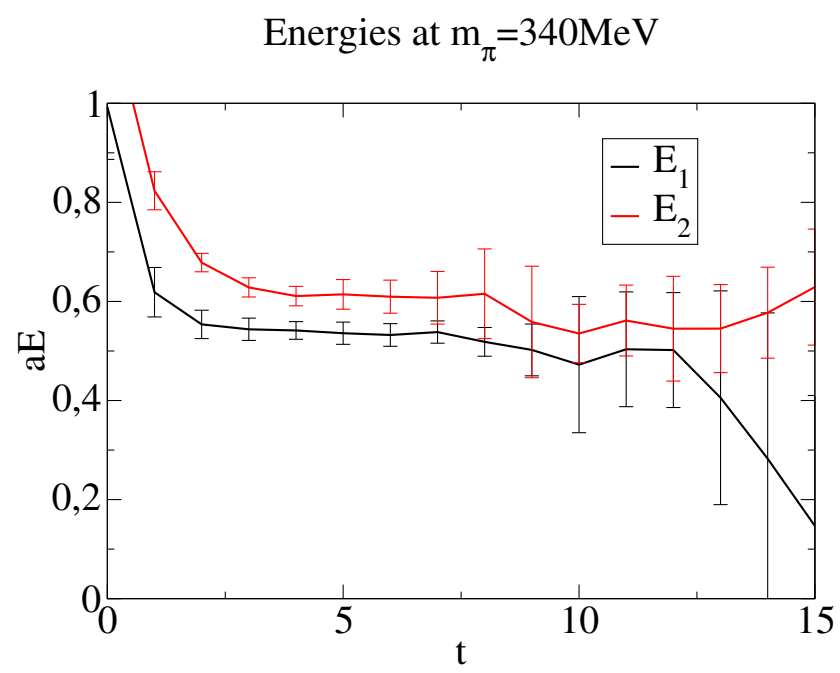

Figure 5: The two energies obtained from solving generalized eigenvalue problem show a clean plateau, and their energy difference is significant. The same goes for $m_{\pi}=200 \mathrm{MeV}$.

from FZ Jülich and from the DECI-5 project HADWIDTH, as well as clusters at Wuppertal and CPT. This work is supported in part by EU grants FP7/2007-2013/ERC n ${ }^{\circ} 208740$, MRTN-CT2006-035482 (FLAVIAnet), OTKA grant AT049652 DFG grant FO 502/2, SFB-TR 55, U.S. Department of Energy Grant No. DE-FG02-05ER25681, by CNRS grants GDR n ${ }^{\circ} 2921$ and PICS $\mathrm{n}^{\circ} 4707$.

\section{References}

[1] S. Durr et al., Science 322 (2008) 1224.

[2] C. Hoelbling, in these proceedings.

[3] S. Aoki et al., Phys. Rev., D76 (2007) 094506.

[4] X. Feng, K. Jansen and D. B. Renner, PoS LAT2009 (2009) 109.

[5] M. Luscher, Commun. Math. Phys. 104 (1986) 177.

[6] M. Luscher, Commun. Math. Phys. 105 (1986) 153.

[7] M. Luscher and U. Wolff, Nucl. Phys. B339 (1990) 222.

[8] M. Luscher, Nucl. Phys. B354 (1991) 531.

[9] M. Luscher, Nucl. Phys. B364 (1991) 237.

[10] S. A. Gottlieb and K. Rummukainen, Nucl. Phys. Proc. Suppl. 47 (1996) 819.

[11] C.H. Kim et al. Nucl. Phys., B727 (2005) 218.

[12] S. Durr et al., arXiv:1011.2403.

[13] S. Durr et al., arXiv:1011.2711.

[14] S. Durr et al., Phys. Rev. D79 (2009) 014501.

[15] T. Kurth et al., arXiv:1011.1780.

[16] M. Luscher and P. Weisz, Commun. Math. Phys. 97 (1985) 59. 